\title{
Optical schemes of spectrographs with a diffractive optical element in a converging beam
}

\author{
E. R. Muslimov \\ eduard.r.muslimov@gmail.com
}

\section{N. K. Pavlycheva}

\begin{abstract}
Kazan National Research Technical University - KAI, Kazan, Tatarstan, Russian Federation, 10 K. Marx str. Kazan, 420111 Russian Federation

Kazan National Research Technical University - KAI, Kazan, Tatarstan, Russian Federation, 10 K. Marx str. Kazan, 420111 Russian Federation
\end{abstract}

Optical schemes of spectrographs based on transmission concave holographic gratings working in converging beams are considered. General description of the design techniques are provided. Each of them is supported by a certain example with calculation and modeling results. In particular, it's shown that combination of such element with a spherical wedge allows to create a spectrograph with correction of astigmatism and a variable-dispersion spectrograph.

[DOI: http://dx.doi.org/10.2971/jeos.2015.15011]

Keywords: Transmission concave holographic grating, converging beam, astigmatism correction, variable dispersion

\section{INTRODUCTION}

Optical schemes of spectrographs with a diffraction grating mounted in a converging beam are known for a long time. They are attractive because of their simplicity and compactness, what explains their use in such fields as astronomy [1,2]. These remarks remain correct also for combinations of gratings and prisms. However, the most of these schemes use plane classical gratings (i.e. gratings with straight and equidistant grooves), which introduce large aberrations and decrease spectral resolution. On the other hand, it's known that concave non-classical gratings have large potential for aberration correction. There are a few examples of use of non-classical aberration-corrected reflection gratings mounted in converging beam for specific tasks [3, 4]. Nevertheless, use of non-classical gratings in such schemes didn't become a self-sufficient optical design tool and, in our opinion, still remains underestimated. Recently the authors have extended the theory of concave grating [5] for a case of transmission concave holographic diffraction grating (TCHDG). It was found, that such optical element has weak optical power and specific properties. A number of schemes based on TCHDG were successfully designed and implemented [6]-[8]. In all of them the grating was mounted in a converging beam. In the present article we discuss advantages of our approach to design of the converging-beam schemes. Further we consider options of extension of the design concept and provide certain examples with necessary calculations and modeling results.

\section{DISCUSSION}

\subsection{Spectrograph with classical grating}

Firstly, we consider a spectrograph scheme with a plane classical transmission grating mounted in a converging beam.
To illustrate the grating aberrations we use a compact spectrograph scheme for the visible range. The spectral working range spreads from 400 to $800 \mathrm{~nm}$. The grating grooves frequency is $400 \mathrm{~mm}^{-1}$. It's imposed on the second surface of a plane-parallel plate made of BK7 glass. We assume that the converging beam is created by an ideal lens $\left(\mathrm{f}^{\prime}=100 \mathrm{~mm}\right.$, $\mathrm{f} / \#=5$ ) set up before the grating and the grating works in normal incidence. The spectrum is detected in the best image plane. The optical scheme drawing is shown on Figure 1. The ray aberrations for this spectrograph scheme are presented in Table 1.

One can see that the grating has huge aberrations including large defocusing and significant astigmatism. This scheme can work only with a wide entrance slit. For instance, if the entrance slit width is $100 \mu \mathrm{m}$, the spectral resolution along the spectrum will change between 3.4 and $4.3 \mathrm{~nm}$. Hereafter we calculate the spectral resolution as product of the spectrograph reciprocal linear dispersion by its instrument function FWHM.

\begin{tabular}{|c|c|cc|cc|cc|}
\hline $\mathrm{m}$ & $\mathrm{M}$ & \multicolumn{2}{|c|}{$\lambda=600 \mathrm{~nm}$} & \multicolumn{2}{c|}{$\lambda=400 \mathrm{~nm}$} & \multicolumn{2}{c|}{$\begin{array}{c}\lambda=800 \mathrm{~nm} \\
\end{array}$} \\
& & \multicolumn{2}{|c|}{$\mathrm{y}^{\prime}=0 \mathrm{~mm}$} & \multicolumn{2}{c|}{$\mathrm{y}^{\prime}=7.90 \mathrm{~mm}$} & $\mathrm{y}^{\prime}=-7.47 \mathrm{~mm}$ \\
\hline & & $\delta \mathrm{z}^{\prime}$ & $\delta \mathrm{y}^{\prime}$ & $\delta \mathrm{z}^{\prime}$ & $\delta \mathrm{y}^{\prime}$ & $\delta \mathrm{z}^{\prime}$ \\
\hline 1.0 & 0 & -436 & 0 & 238 & 0 & -413 & 0 \\
-0.5 & 0 & -123 & 0 & -53 & 0 & -86 & 0 \\
0.5 & 0 & -58 & 0 & -70 & 0 & -151 & 0 \\
1.0 & 0 & -292 & 0 & -255 & 0 & -538 & 0 \\
0 & 0.5 & -28 & 282 & -20 & 105 & -35 & 422 \\
0 & 1.0 & -112 & 568 & -80 & 214 & -142 & 849 \\
\hline
\end{tabular}

TABLE 1 Aberrations of the spectrograph with plane classical grating. 


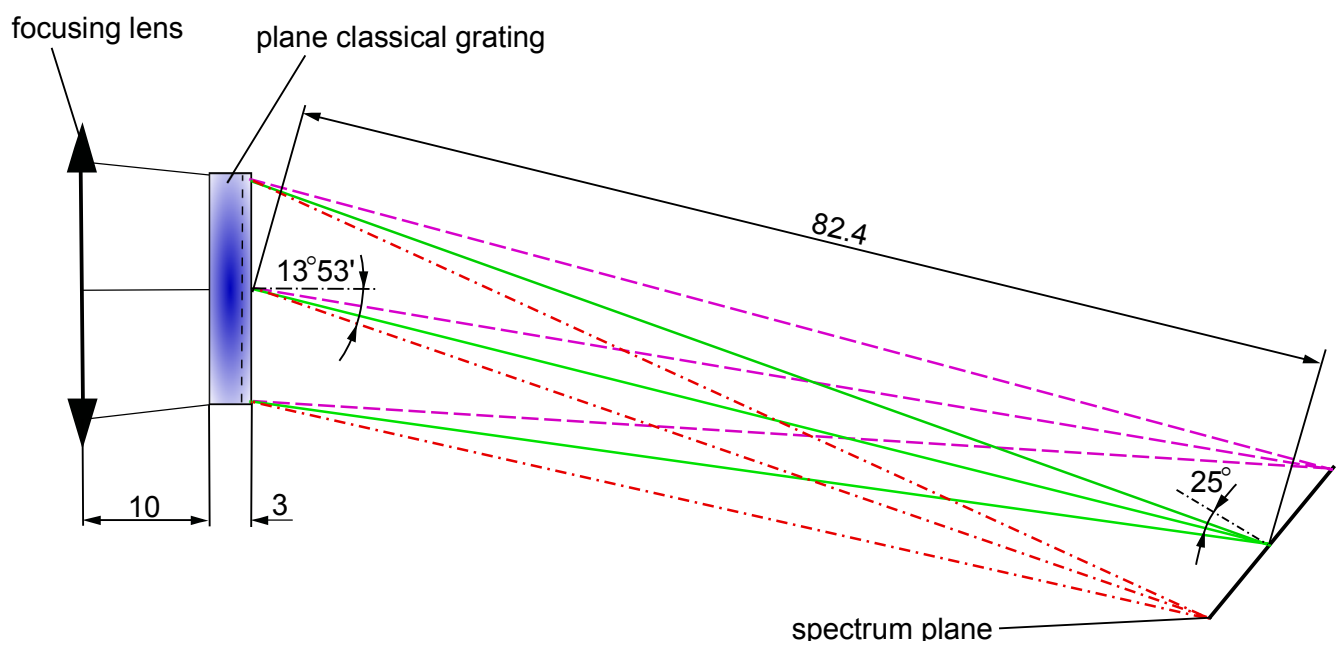

FIG. 1 Optical scheme of the spectrograph with plane classical grating.

\subsection{Flat-field spectrograph with transmission concave holographic grating}

The method of design of a flat-field spectrograph scheme with TCHDG is based on analytical minimization of the grating aberration function terms, which was used, for example, in [9] and [10]. This approach allows to construct a simple design technique, which provides obtaining of appropriate optical scheme for a wide range of initial parameters. The analytical design solution can be either used directly, or serve as a starting point for numerical optimization of the scheme. We must note that the approach has some theoretical limitations, restricting its use for design of high-aperture and highdispersion schemes [11]. However in the most cases of practical interest large values of aperture and dispersion aren't achievable because of aberrations of the transmission grating and its substrate. Focusing and aberration properties of the grating can be described by its aberration function, which is series expansion of optical path function for a ray emitted from the entrance slit center and diffracted in an arbitrary point on the grating surface.

$$
\begin{aligned}
V= & -y F_{0}+\frac{y^{2}}{2 R} F_{1}+\frac{z^{2}}{2 R} F_{2}+\frac{y^{3}}{2 R^{2}} F_{3}+\frac{y z^{2}}{2 R^{2}} F_{4}+\frac{y^{4}}{8 R^{3}} F_{5} \\
& +\frac{y^{2} z^{2}}{4 R^{3}} F_{6}+\frac{z^{4}}{8 R^{3}} F_{7}+\ldots
\end{aligned}
$$

Here $R$ is the grating surface radius, $(z, y)$ are Cartesian coordinates of the diffraction point and $F_{i}$ are coefficients, defining certain aberrations (tangential defocusing, astigmatism, tangential coma, sagittal coma, spherical aberrations etc.). We assume that the coefficients change across the grating surface is negligible. If we consider a $1^{\text {st }}$ generation holographic grating (i.e. grating recorded with 2 coherent point sources [12]) with defined grooves frequency, there are 3 free parameters for aberration correction. So it's possible to correct defocusing along the spectrum as well as tangential coma and astigmatism in its center. For considerations of simplicity we suppose that the spectrum plane is orthogonal to the chief ray on the medium wavelength. Then the conditions of aberration cor- rection can be written as follows

$$
\begin{gathered}
a_{1} \frac{R}{6 d_{a v}^{\prime}}+a_{2}-a_{3} \frac{H_{1}}{N \lambda_{0}}=0, \\
-a_{3} \frac{R}{d_{a v}^{\prime}}-a_{4}+a_{5} \frac{H_{1}}{N \lambda_{0}}=0, \\
R\left[\left(\frac{1}{d}+\frac{\cos \varphi}{R}\right)+\left(\frac{1}{d_{a v}^{\prime}}-\frac{\cos \varphi_{a v}^{\prime}}{R}\right)\right]-\frac{k \lambda_{a v}}{\lambda_{0}} H_{2}=0, \\
R^{2}\left[\frac{\sin \varphi}{d}\left(\frac{\cos \varphi}{R}+\frac{\cos ^{2} \varphi}{d}\right)+\frac{\sin \varphi_{a v}^{\prime}}{d_{a v}^{\prime}}\right. \\
\left.\cdot\left(-\frac{\cos \varphi_{a v}^{\prime}}{R}+\frac{\cos ^{2} \varphi_{a v}^{\prime}}{d_{a v}^{\prime}}\right)\right]-\frac{k \lambda_{a v}}{\lambda_{0}} H_{3}=0 .
\end{gathered}
$$

where

$$
\begin{aligned}
a_{1}= & \cos 2 \varphi_{a v}^{\prime} \sin \varphi^{\prime} \cos ^{5} \varphi^{\prime}-\sin 2 \varphi_{a v}^{\prime} \cos ^{6} \varphi^{\prime} \\
& +\left(5 \cos 2 \varphi_{a v}^{\prime}+6 \sin ^{2} \varphi_{a v}^{\prime}\right) \\
& \cdot\left(\frac{3}{8} \varphi^{\prime}+\frac{1}{4} \sin 2 \varphi^{\prime}+\frac{1}{32} \sin 4 \varphi^{\prime}\right), \\
a_{2}= & S \cos \varphi_{a v}^{\prime}\left(\sin \varphi-\frac{1}{3} \sin ^{3} \varphi^{\prime}\right)-S \sin \varphi_{a v}^{\prime} \frac{\cos ^{3} \varphi^{\prime}}{3} \\
& -\cos \varphi_{a v}^{\prime}\left(\frac{3}{8} \varphi^{\prime}+\frac{1}{4} \sin 2 \varphi^{\prime}+\frac{1}{32} \sin 4 \varphi^{\prime}\right) \\
& +\sin \varphi_{a v}^{\prime} \frac{\cos ^{4} \varphi^{\prime}}{4}, \\
a_{3}= & -\cos \varphi_{a v}^{\prime} \frac{\cos ^{4} \varphi^{\prime}}{4}+\sin _{a v}^{\prime}\left(\frac{\varphi^{\prime}}{8}-\frac{\sin 4 \varphi^{\prime}}{32}\right) \\
& -\sin \varphi \cos \varphi_{a v}^{\prime}\left(\sin ^{\prime}-\frac{1}{3} \sin ^{3} \varphi^{\prime}\right) \\
& +\sin \varphi \sin \varphi_{a v}^{\prime} \frac{\cos ^{3} \varphi_{a v}^{\prime}}{3}, \\
a_{4}= & -S \cos \varphi^{\prime}-S \sin \varphi^{\prime}-\frac{\sin ^{2} \varphi^{\prime}}{2}+\sin \varphi \sin \varphi^{\prime}, \\
a_{5}= & \frac{1}{2} \varphi^{\prime}-\frac{\sin 2 \varphi^{\prime}}{4}+\frac{1}{2} \sin ^{2} \phi^{\prime}+2 \sin \varphi \cos \varphi^{\prime}, \\
S= & \frac{R \cos { }^{2} \varphi}{d}+\cos ^{\prime} \varphi^{\prime}
\end{aligned}
$$

The notations here are: $(d, \varphi)$ are polar coordinates of the entrance slit center, $\left(d^{\prime}, \varphi^{\prime}\right)$ are coordinates of its monochromatic image, $\lambda$ is the working wavelength, $\lambda_{0}$ is the recording wavelength, $N$ is the grooves frequency, $k$ is the diffraction order and $H_{i}$ are holographic coefficients similar to those used in [5] 


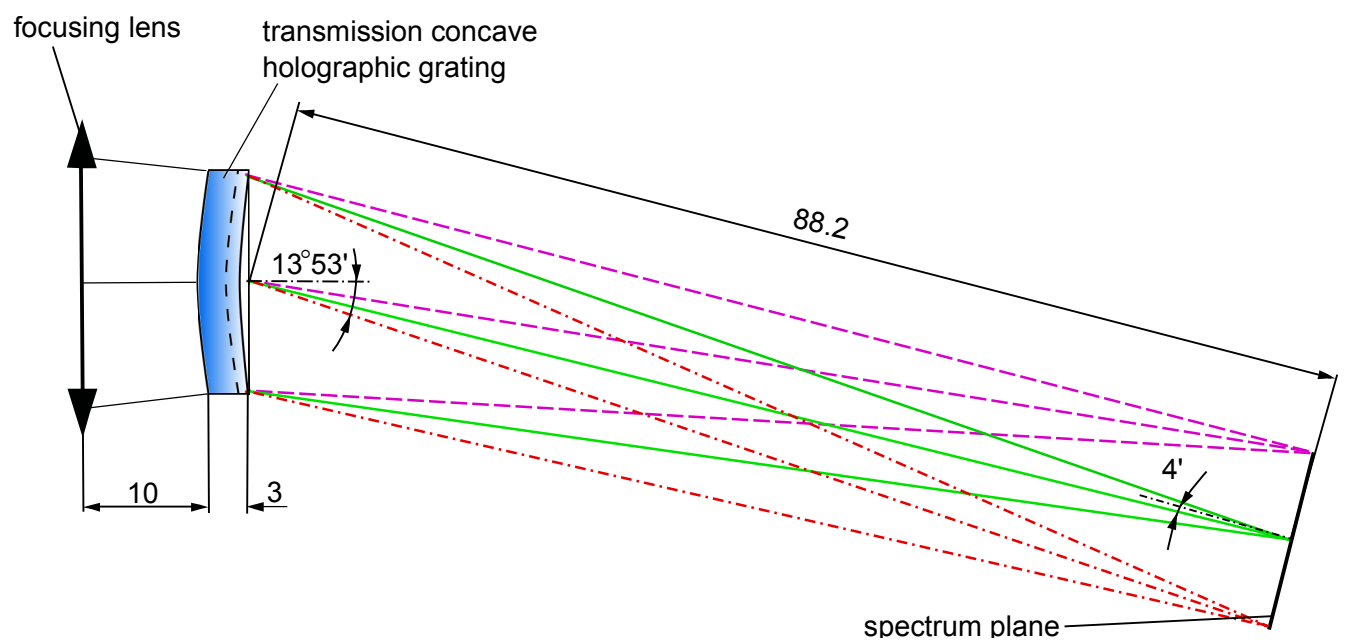

FIG. 2 Optical scheme of the flat-field spectrograph with TCHDG.

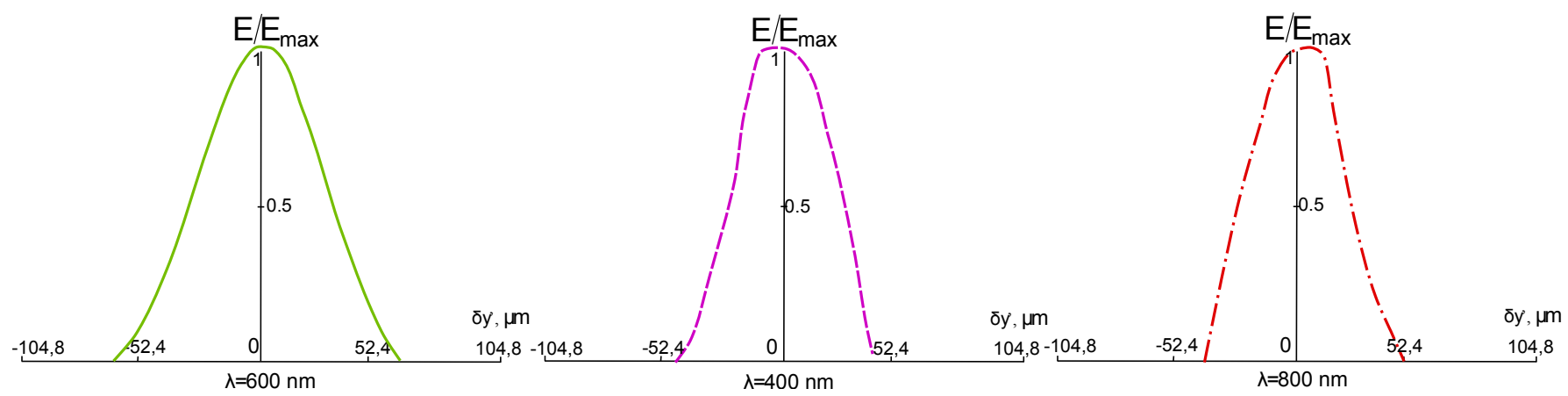

FIG. 3 Instrument functions of the flat-field spectrograph.

\begin{tabular}{|c|c|cc|cc|cc|}
\hline $\mathrm{m}$ & $\mathrm{M}$ & \multicolumn{2}{|c|}{$\lambda=600 \mathrm{~nm}$} & \multicolumn{2}{c|}{$\lambda=400 \mathrm{~nm}$} & \multicolumn{2}{c|}{$\lambda=800 \mathrm{~nm}$} \\
& & \multicolumn{2}{|c|}{$\mathrm{y}^{\prime}=0 \mathrm{~mm}$} & \multicolumn{2}{c|}{$\mathrm{y}^{\prime}=7.90 \mathrm{~mm}$} & $\mathrm{y}^{\prime}=-7.47 \mathrm{~mm}$ \\
\hline & & $\delta \mathrm{y}^{\prime}$ & $\delta \mathrm{z}^{\prime}$ & $\delta \mathrm{y}^{\prime}$ & $\delta \mathrm{z}^{\prime}$ & $\delta \mathrm{y}^{\prime}$ & $\delta \mathrm{z}^{\prime}$ \\
\hline 1.0 & 0 & -38 & 0 & 13 & 0 & 23 & 0 \\
-0.5 & 0 & -19 & 0 & 8 & 0 & 11 & 0 \\
0.5 & 0 & -18 & 0 & -10 & 0 & -8 & 0 \\
1.0 & 0 & -34 & 0 & -23 & 0 & -12 & 0 \\
0 & 0.5 & -0 & 12 & 0 & -69 & 0 & 93 \\
0 & 1.0 & -1 & 24 & -2 & -137 & 1 & 187 \\
\hline
\end{tabular}

TABLE 2 Aberrations of the flat-field spectrograph with TCHDG.

and [10], while " $a v$ " subscript marks values relating to the average wavelength of the working range. Expressions denoted in Eqs. (2) and (3) are also derived easily from the equations shown in [9]. By solving system (2) one can find the spectrum position and holographic coefficients, from which coordinates of recording sources can be defined. Let's consider a flat-field scheme similar to that described in the previous section. Again we have a grating with grooves frequency of $400 \mathrm{~mm}^{-1}$, placed $10 \mathrm{~mm}$ behind the ideal lens $\left(\mathrm{f}^{\prime}=100 \mathrm{~mm}\right.$, $\mathrm{f} / \#=5$ ). The spectrograph working spectral range is 400 $800 \mathrm{~nm}$. In this case the grating substrate is achromatic meniscus made of BK7 glass. The center of its second surface coincides with the incident beam focus. Using Eqs. (2)-(3) we find coordinates of the spectrum center - $\left(89.15 \mathrm{~mm}, 13^{\circ} 53^{\prime}\right)$. The holographic coefficients are $H_{1}=-0.03906, H_{2}=0.00375$, $H_{3}=0.00881$. The corresponding coordinates of recording point sources for $\lambda_{0}=441.6 \mathrm{~nm}$ (He-Cd laser) are $(88.94 \mathrm{~mm}$, $\left.12^{\circ} 15^{\prime}\right)$ and $\left(87.30 \mathrm{~mm}, 2^{\circ} 2^{\prime}\right)$. The spectrograph optical scheme after definition of the best image plane is shown on Figure 2.
To evaluate the spectrograph image quality we consider its geometrical aberrations on the main wavelengths (Table 2). It's clear from the table that good focusing on the plane along all the working range is obtained, while the tangential coma and astigmatism for the central wavelength are almost corrected. For detailed investigation of the spectral resolution we consider the spectrograph instrument functions (Figure 3). The entrance slit width is $50 \mu \mathrm{m}$.

The instrument function FWHMs at 600, 400 and $800 \mathrm{~nm}$ are $62.4,50$ and $50 \mu \mathrm{m}$, respectively. Accounting for the reciprocal linear dispersion, which is equal to $27.4 \mathrm{~nm} / \mathrm{mm}$, we find that the corresponding values of spectral resolution are 1.7, 1.4 and $1.4 \mathrm{~nm}$. Thus the flat-field spectrograph scheme provides quite a high spectral resolution. In addition such features of the scheme as good astigmatism correction, relatively small deviation angle and weak focusing properties of the grating should be emphasized. In the following sections we propose new scheme designs using these properties of the transmission concave grating.

\subsection{Astigmatism-corrected spectrograph}

As it was shown above, the spectrograph scheme based on TCHDG mounted in a converging beam is notable for its low astigmatism. The astigmatism can be completely corrected in the spectrum center, but it slowly increases towards the edges. This phenomenon can be explained by a tilt between tangential and sagittal focal curves, which intersect each other in the spectrum center. A simple way to reduce this tilt is introducing of a spherical wedge into the optical scheme. We assume 
that the first surface of the wedge coincides with the grating surface. The obtained optical component, which represents a combination of grating and wedge (prism) is usually called grism. Such elements are widely used in imaging and multichannel spectrometers [13]-[15].

The design procedure consists of several stages. During the first stage, the grating parameters are defined from aberration correction equations, similar to Eqs. (2)-(3). In contrast with that system, properties of the grism material must be accounted for. So the equations take on the following form:

$$
\begin{aligned}
& \frac{\partial \sum_{j=1}^{p}}{\partial d_{a v}^{\prime}}\left[n_{1 j}\left(-\frac{R \cos ^{2} \phi}{d}+\cos \phi\right)\right. \\
& \left.+n_{2 j}\left(-\frac{R \cos ^{2} \phi_{j}^{\prime}}{d_{j}^{\prime}}-\cos \phi_{j}^{\prime}\right)-H_{1} \frac{k \lambda_{j}}{\lambda_{0}}\right]^{2}=0, \\
& \partial \sum^{p} \\
& \frac{\partial \sum_{j=1}}{\partial H_{1}}\left[n_{1 j}\left(-\frac{R \cos ^{2} \phi}{d}+\cos \phi\right)\right. \\
& \left.+n_{2 j}\left(\frac{R \cos ^{2} \phi_{j}^{\prime}}{d_{j}^{\prime}}-\cos \phi_{j}^{\prime}\right)-H_{1} \frac{k \lambda_{j}}{\lambda_{0}}\right]^{2}=0, \\
& H_{2}=\frac{k \lambda_{0}}{\lambda_{a v}}\left[\left(-\frac{R}{d}+\cos \varphi\right)+n_{a v}\left(\frac{R}{d_{a v}^{\prime}}-\cos \varphi_{a v}^{\prime}\right)\right] \text {, } \\
& H_{3}=-\frac{k \lambda_{0}}{\lambda_{a v}}\left[\frac{R^{2} \sin \varphi}{d}\left(-\frac{\cos ^{2} \varphi}{d}+\frac{\cos \varphi}{R}\right)\right. \\
& \left.-n_{a v} \frac{R^{2} \sin \varphi_{a v}^{\prime}}{d_{a v}^{\prime}}\left(\frac{\cos ^{2} \varphi_{a v}^{\prime}}{d_{a v}^{\prime}}-\frac{\cos \varphi_{a v}^{\prime}}{R}\right)\right] \text {. }
\end{aligned}
$$

Here $n_{1}$ and $n_{2}$ are refraction indexes for media before and after the grating, respectively. The focal curves for the grating in tangential and sagittal planes are defined by

$$
\begin{gathered}
d_{t}^{\prime}=\frac{n \cos ^{2} \varphi^{\prime}}{\frac{\cos ^{\prime} \varphi}{d}+\frac{n \cos \varphi^{\prime}-\cos \varphi}{R}-\frac{\sin \varphi-n \sin \varphi^{\prime}}{R N \lambda_{0}} H_{1}} \\
d_{s}^{\prime}=\frac{n}{\frac{1}{d}+\frac{n \cos \varphi^{\prime}-\cos \varphi}{R}-\frac{\sin \varphi-n \sin \varphi^{\prime}}{R N \lambda_{0}} H_{2}}
\end{gathered}
$$

On the second stage we introduce a spherical wedge into the system. We suppose that its angle $\psi$ and second surface radius $R_{1}$ are variable and axial thickness $t$ is fixed. The astigmatic difference in the image after wedge is

$$
\begin{aligned}
x_{t}^{\prime} & -x_{s}^{\prime} \\
& =\frac{R_{1} \tilde{d}_{t}^{\prime} \cos \tilde{\varphi}^{\prime}}{(1-n) \tilde{d}_{t}^{\prime} \cos \tilde{\varphi}^{\prime}+R_{1} n}-\frac{R_{1} \tilde{d}_{s}^{\prime} \cos ^{2} \tilde{\varphi}^{\prime}}{(1-n) \tilde{d}_{s}^{\prime}+\cos \tilde{\varphi}^{\prime} R_{1} n}
\end{aligned}
$$

Here $\left(\tilde{d}^{\prime}, \tilde{\varphi}^{\prime}\right)$ are coordinates of the image point after the wedge. The wedge parameters can be found by means of optimization of a simple merit function:

$$
f_{\text {ast }}\left(R_{1}, \psi\right)=\sum_{g=1}^{q}\left[\left(x_{t}\left(R_{1}, \psi, \lambda_{g}\right)-x_{s}\left(R_{1}, \psi, \lambda_{g}\right)\right) R_{1}\right]^{2}
$$

On the final stage numerical optimization of the scheme in whole is performed. Because the deviation angles for the grating and wedge are relatively small and have different signs, after the optimization the scheme can be made axial.
To exemplify the design algorithm, let's consider a scheme of astigmatism-corrected spectrograph for the visible domain. The working spectral range is $400-700 \mathrm{~nm}$. The grism is mounted in normal incidence after an ideal lens $\left(\mathrm{f}^{\prime}=160 \mathrm{~mm}\right.$, $\mathrm{f} / \#$ = 7.5). Initial distance from the incidence beam focus to the grating is $150 \mathrm{~mm}$ (it's equal to the grating radius), and initial grating grooves frequency is $450 \mathrm{~mm}^{-1}$. Both of the grating substrate and wedge are made of BK7 glass. According to the described procedure we find the coordinates of spectrum center $\left(151.78 \mathrm{~mm}, 9^{\circ} 23^{\prime}\right)$ and the holographic coefficients $H_{1}=-0.03110, H_{2}=0.001983, H_{3}=0.00484$. The recording point sources coordinates for $\lambda_{0}=441.6 \mathrm{~nm}$ are $\left(151.62 \mathrm{~mm}, 10^{\circ} 34^{\prime}\right)$ and $\left(149.35 \mathrm{~mm},-52^{\circ} 17^{\prime}\right)$. On the second stage by using of conjugate gradient method we minimize function (8) and obtain the wedge parameters: $R_{1}=142.115 \mathrm{~mm}, \psi=13^{\circ} 18^{\prime}$. Coordinates of the spectrum center after the wedge are $\left(149.21 \mathrm{~mm}, 7^{\circ} 49^{\prime}\right)$.

To illustrate the achieved astigmatism correction we consider the focal curves in tangential and sagittal planes (Figure 4). The right plot corresponds to the spectrograph scheme with grism and the left plot corresponds to an equivalent scheme with single transmission grating.

As it is seen on the plots, the focal curves tilt is significantly decreased. It also can be noted that the deviation angle is smaller. After numerical optimization of the scheme we obtain final design with the following parameters: the grating surface radius $69.50 \mathrm{~mm}$, the radius of grating substrate first surface $86.12 \mathrm{~mm}$, the grating recording parameters $\left(97.93 \mathrm{~mm}, 9^{\circ} 17^{\prime}\right)$ and $\left(96.18 \mathrm{~mm},-1^{\circ} 43^{\prime}\right)$.

The final view of the optical scheme is presented on Figure 5.

Figure 6 shows the spectrograph instrument functions (the entrance slit width is $15 \mu \mathrm{m})$. The FWHM values for the spectrum center and its edges are 15.0, 15.9 and $15.0 \mu \mathrm{m}$. As soon as the reciprocal linear dispersion is $15 \mathrm{~nm} / \mathrm{mm}$, the spectral resolution is $0.23,0.24$ and $0.23 \mathrm{~nm}$.

On Figure 7 the spectrograph spot diagrams are provided to demonstrate the astigmatism correction obtained in the final scheme. The transverse dimensions of the diagrams are 19.1-34.1 $\mu \mathrm{m}$.

\subsection{Variable-dispersion spectrograph}

In some cases it would be very attractive to change spectrometer dispersion and spectral resolution during measurement. There are a few examples of such spectral instruments, but they use complicated zoom optical systems to change the dispersion [16].

Properties of the optical schemes considered above suggest that it's possible to build a simple variable-dispersion spectrograph on the basis of TCHDG. In such spectrograph the grating is mounted into a converging beam. Weak focusing properties of the grating together with its aberration correction capabilities allow to find at least two positions of the grating, in which different spectral ranges are focused on the same plane. In this case the grating, and therefore, its angular dispersion 

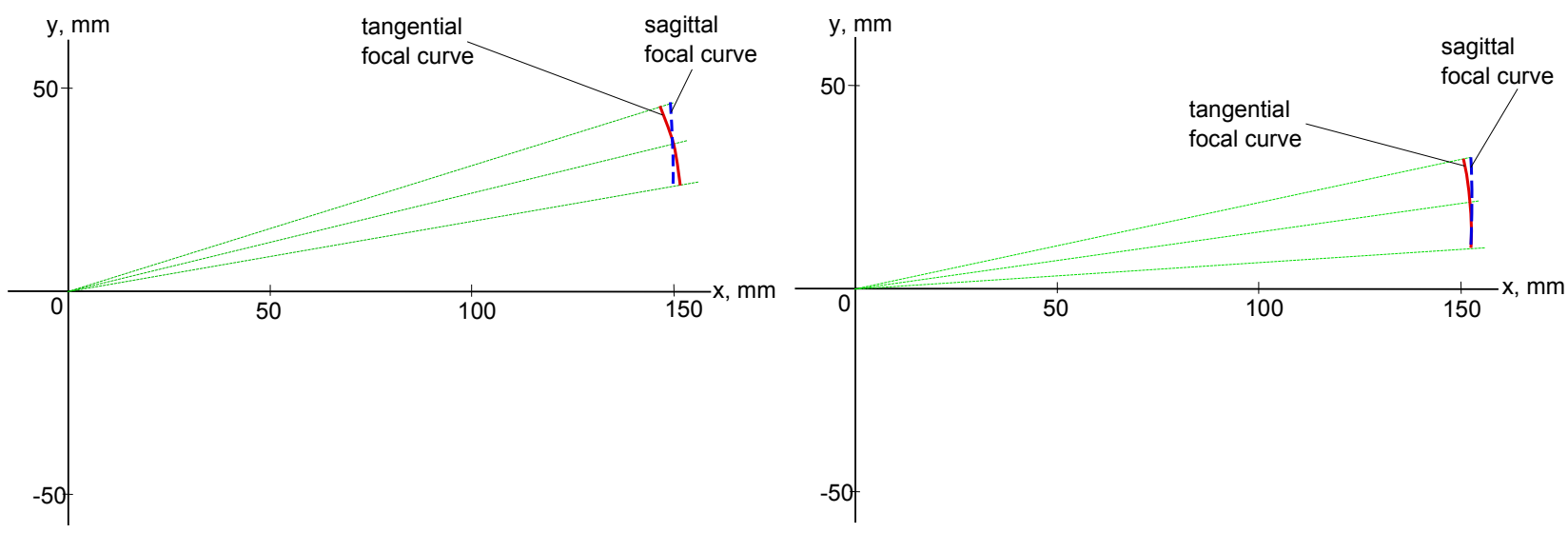

FIG. 4 Focal curves of the spectrographs. Left: single grating spectrograph; Right: grism spectrograph.

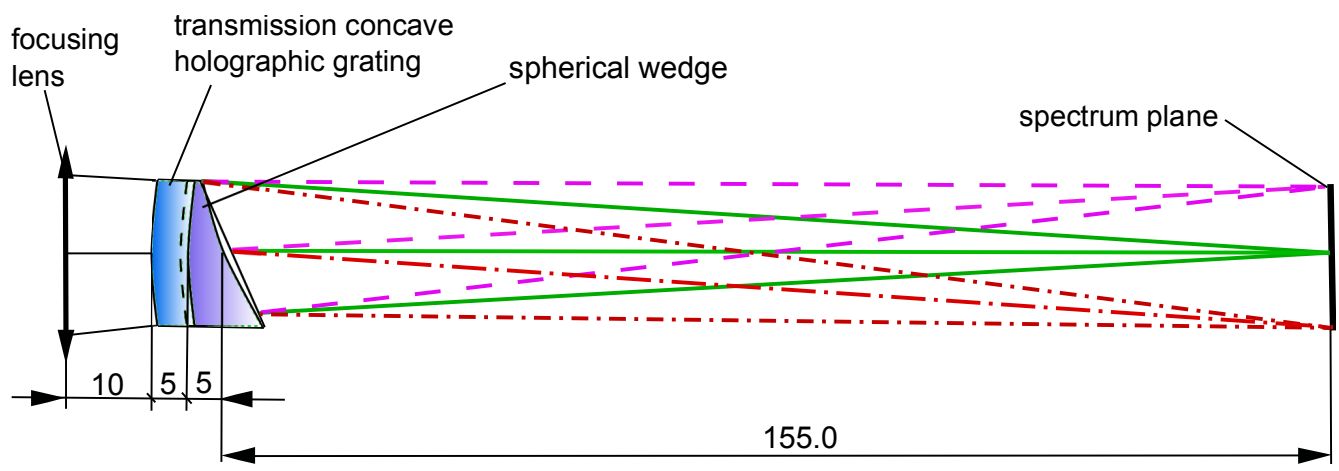

FIG. 5 Optical scheme of the astigmatism corrected spectrograph.
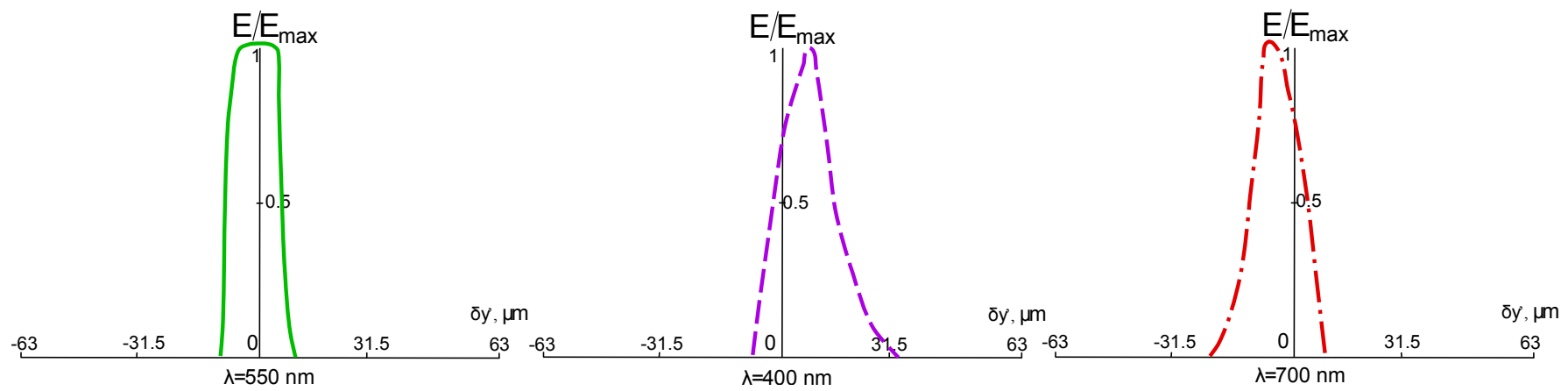

FIG. 6 Instrument functions of the astigmatism-corrected spectrograph.

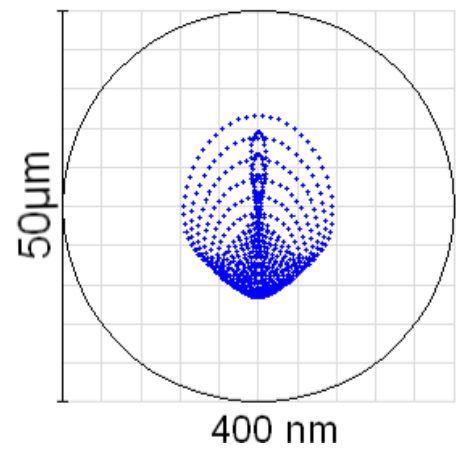

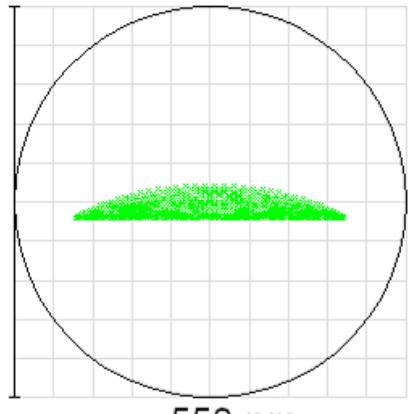

$550 \mathrm{~nm}$

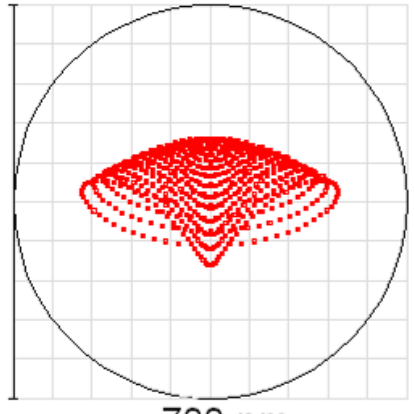

$700 \mathrm{~nm}$

FIG. 7 Spot diagrams of the astigmatism-corrected spectrograph.

remain the same as well as the length of the detector, while the linear dispersion and spectral resolution change. The working ranges are centred around one wavelength. As we could see before, introducing of a spherical wedge allows to implement an additional aberration correction and make the scheme completely axial. The latter property could reduce the dispersion change to simple translation of the diffractive optical component.

To show feasibility of such design and find an initial point for it, let's examine dependence of the TCHDG holographic coefficients on the position of the incident beam focus. We 
calculated parameters of a number of flat-field spectrograph schemes with the same spectrum length, grating radius and grooves frequency. The positions of incident beam focus and spectrum as well as the working range were different. For each of them we solved Eqs. (2)-(3) and found the holographic coefficients. The obtained dependencies of normalized values of $H_{1}-H_{3}$ on the distance to beam focus normalized to the grating radius are shown on Figure 8 .

It can be seen from the graphs that values of $H_{1}$ and $H_{3}$ corresponding to aberration correction vary only slightly for distances more than $R$. It means that the tangential terms of aberrations can be corrected in different positions of the same grating. The astigmatism changes rapidly and can't be reduced, so further it can be removed from the correction conditions. The initial point corresponds to the longer distance to the focus and wider spectral range. The grating parameters are calculated from Eqs. (2)-(3).

As soon as the detector length in different position remains the same, an obvious condition should be met:

$$
d^{\prime} \tan \left(\arcsin k \lambda N-\varphi_{a v}^{\prime}\right)=\text { const. }
$$

The wedge angle is determined by axial arrangement of the

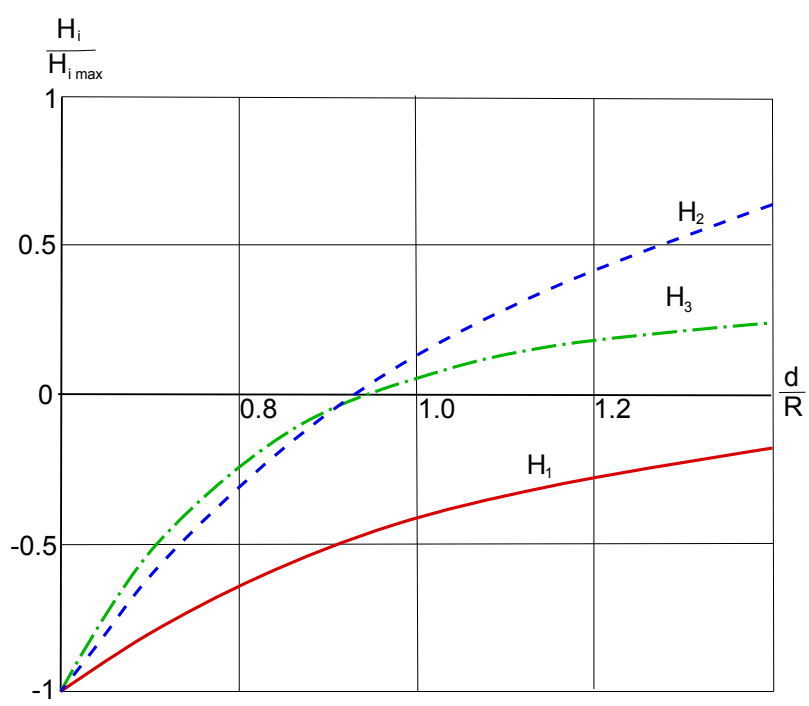

FIG. 8 Dependencies of holographic coefficients on the focus position. scheme:

$$
\psi=\arctan \left(\frac{n \sin \varphi_{a v}^{\prime}}{n \sin \varphi_{a v}^{\prime}-1}\right)
$$

The wedge second surface is assumed to have the same radius as the grating. After this rough definition of the starting point, the scheme can be optimized numerically. During the optimization the positions of lens and detector as well as the spectrum length should be maintained. As we mentioned before, the astigmatism can be except. As an example of this approach we consider a variable-dispersion spectrograph scheme for the range of $400-800 \mathrm{~nm}$. In this scheme a movable grism is mounted after a specially designed projection lens $\left(f^{\prime}=90.5 \mathrm{~mm}, \mathrm{f} / \#=6.5\right)$. An aspheric field lens is used to correct residual aberrations. In the first position spectrum for the full range of $400-800 \mathrm{~nm}$ is focused on the detector with reciprocal linear dispersion of $30.7 \mathrm{~nm} / \mathrm{mm}$. When the grism moves to the second position, the central part of the spectrum $500-700 \mathrm{~nm}$ is projected onto the same detector with dispersion of $15.4 \mathrm{~nm} / \mathrm{mm}$. Both positions of the scheme are presented on Figure 9.

The corresponding instrument functions are presented on Figure 10. For the first position the spectral resolution is $1.5-1.9 \mathrm{~nm}$, and in the second one it reaches $0.7 \mathrm{~nm}$.

Thus the designed spectrograph scheme works in two positions with moderately high spectral resolution. The spectrograph is notable for its compactness and extremely simple mechanical scheme. The preliminary estimates show that the found solution can be extended for a multi-position design and also can be applied for other combinations of working spectral ranges.

\section{CONCLUSIONS}

Thus in the presented work we proposed a group of new spectrograph optical schemes and approaches to their design. All of the schemes are built on the basis of a diffractive optical element, mounted in a converging beam. Such setup is very simple, compact and allows to create a direct-vision scheme. It was shown that a transmission concave holographic grating (TCHDG) can be used in a flat-field spectrograph scheme.

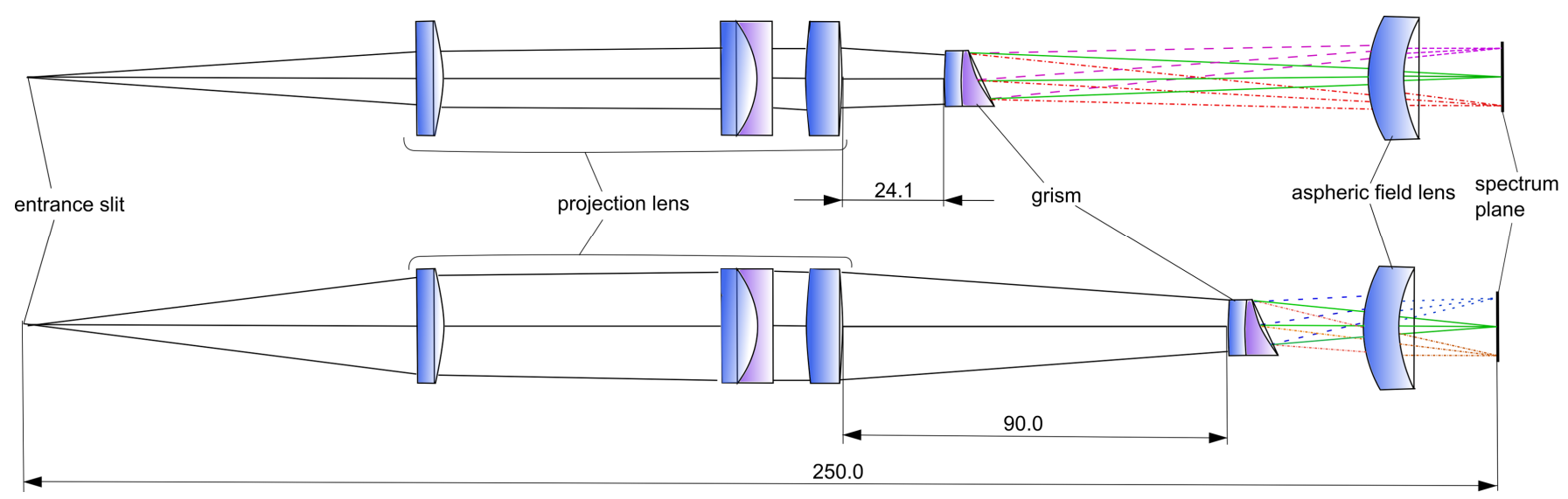

FIG. 9 Optical scheme of the variable dispersion spectrograph. Top: position for working in the 400-800 nm range; Bottom: position for working in the 500-700 nm range. 


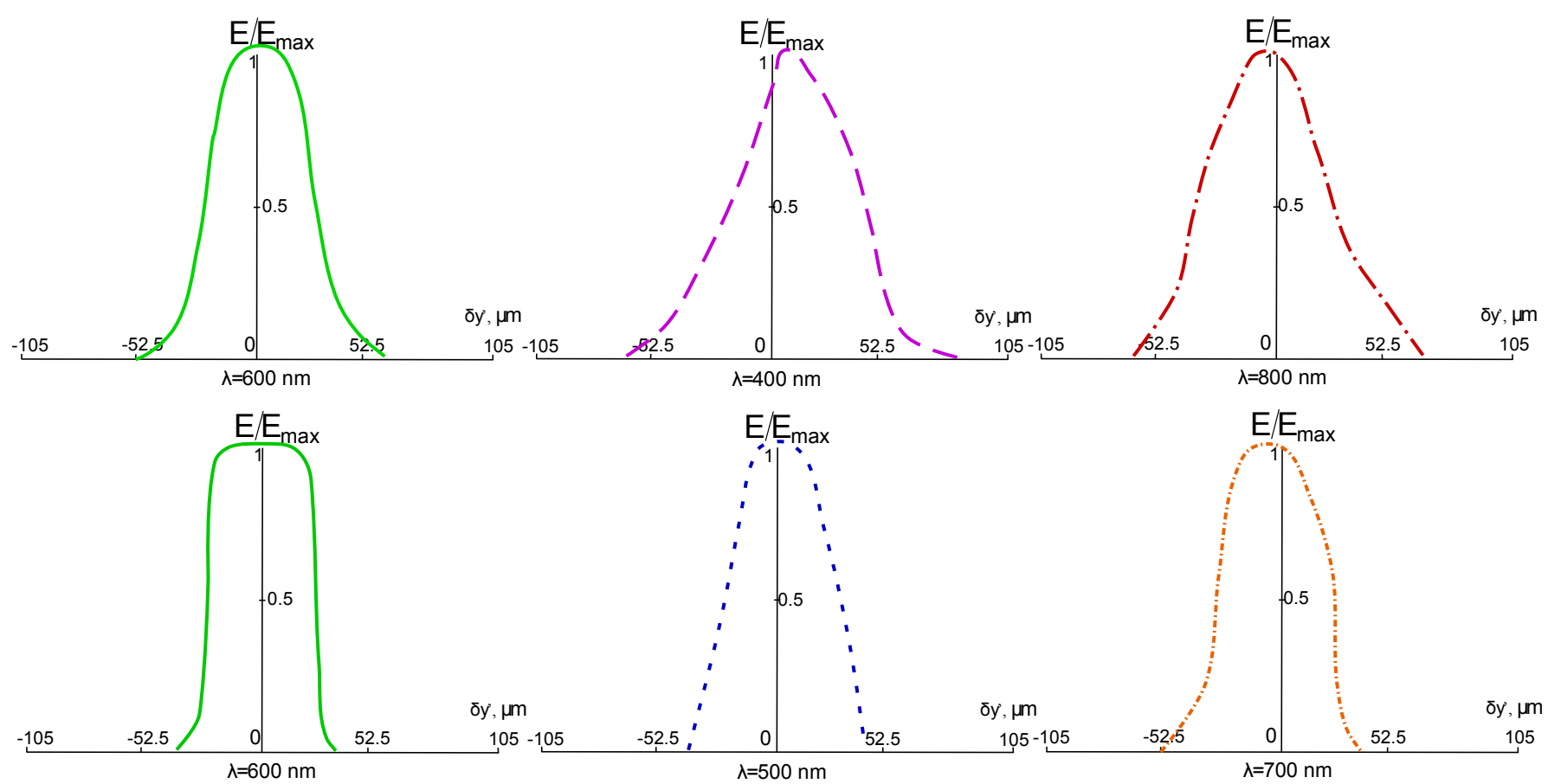

FIC. 10 Instrument functions of the variable-dispersion spectrograph.

It provides relatively high spectral resolution (up to 4 times better in comparison with a classical grating) and has such features as low astigmatism, small deviation angle and weak optical power. Two optical schemes of spectrographs using these specific features are proposed. In the first one a combination of TCHDG and spherical wedge - grism is used to correct the astigmatism along the spectrum. The scheme is characterized by axial arrangement and high image quality. In the second design the grism is moved to obtain different linear dispersion with two spectral working ranges. The mechanical scheme of the variable-dispersion spectrograph is extremely simple, while the spectral resolution in the both of positions is relatively high. We think that the developed optical schemes and corresponding design algorithms (analytical or semi-analytical) open prospects of creation of new spectral devices with extended functionalities and enhanced performance.

\section{ACKNOWLEDGEMENTS}

The authors would like to thank their colleagues from State Institute of Applied Optics (Kazan) for help with modelling of the optical schemes.

\section{References}

[1] K. H. Nordsieck, A. D. Code, and C. M. Anderson, "Exploring ultraviolet astronomical polarimetry: results from the Wisconsin Ultraviolet Photo-Polarimeter Experiment (WUPPE)," Proc. SPIE 2010, 2-11 (1993).

[2] K. M. Harrison, Grating spectroscopes and how to use them (Springer, Berlin, 2012).

[3] A. V. Savushkin, "Design of stigmatic gratings for grazing incidence monochromator spectrographs," Proc. SPIE 2805, 169-174 (1996).
[4] E. Wilkinson, and J. C. Green, "First-generation holographic, grazing-incidence gratings for use in converging, extremeultraviolet light beams," Appl. Opt. 34, 4685-4696 (1995).

[5] T. Namioka, "Theory of the concave grating," J. Opt. Soc. Am. 49, 446-460 (1959).

[6] N. K. Pavlycheva, and E. R. Muslimov, "Compact dual-band spectrograph," Adv. Opt. Technol. 1, 455-461 (2012).

[7] E. R. Muslimov, "Transmission holographic grating with improved diffraction efficiency for a flat-field spectrograph," Proc. SPIE 8787, 87870B (2012).

[8] E. Muslimov, Optical schemes of spectrographs with transmission concave holographic gratings (2013 CIOMP-OSA Summer Session on Optical Engineering, Design and Manufacturing, Changchun, August 4-9, 2013).

[9] H. Noda, T. Namioka, and M. Seya, "Geometric theory of the grating," J. Opt. Soc. Am. 64, 1031-1036 (1974).

[10] M. M. Nazmeev, and N. K. Pavlycheva, "New generation spectrographs," Opt. Eng. 33, 2777-2782 (1994).

[11] T. Namioka, M. Koike, and D. Content, "Geometric theory of the ellipsoidal grating," Appl. Opt. 33, 7261-7274 (1994).

[12] C. Palmer, and E. Loewen, Diffraction grating handbook (sixth edition, Newport Corp., Rochester, 2005).

[13] M. Aikio, Hyperspectral prism-grating-prism imaging spectrograph (PhD thesis, VTT Technical Research Centre of Finland, 2001).

[14] 0. Pawluczyk, and R. Pawluczyk, "Applications of multichannel imaging spectrometer," Proc. SPIE 5578, 227-238 (2004).

[15] T. Hyvarinen, E. Herrala, and A. Dall'Ava, "Direct sight imaging spectrograph: a unique add-on component brings spectral imaging to industrial applications," Proc. SPIE 3302, 3302-3321 (1998).

[16] J. Choi, T. H. Kim, H. J. Kong, and J. U. Lee, "Zoom lens design for a novel imaging spectrometer that controls spatial and spectral resolution individually," Appl. 0pt. 45, 3430-3441 (2006). 